\title{
Reviewers of the current issue
}

(BIRDEM Med J 2021; 11(3): 94-253)

1. Prof. Abid Hossain Mollah

Professor

Department of Paediatrics

BIRDEM General Hospital, Dhaka, Bangladesh

\section{Prof. Ferdousi Begum}

Professor

Department of Obstetrics and Gynaecology

BIRDEM General Hospital, Dhaka, Bangladesh

3. Prof. Masuda Mohsena

Professor

Department of Community Medicine

Ibrahim Medical College, Dhaka, Bangladesh

4. Dr. Mohammad Kamrul Ahsan

Associate Professor

Department of Dermatology

BIRDEM General Hospital, Dhaka, Bangladesh

5. Dr. Tabassum Samad

Associate Professor

Department of Nephrology

BIRDEM General Hospital, Dhaka, Bangladesh

6. Dr. Mohammad Aminur Rahman

Assistant Professor

Department of Neurology

Sir Salimullah Medical College, Dhaka, Bangladesh

\section{Dr. Rumana Habib}

Associate Professor

Department of Neurology

BIRDEM General Hospital, Dhaka, Bangladesh
8. Dr. Wasim Md. Mohosin Ul Haque

Associate Professor

Department of Nephrology

BIRDEM General Hospital, Dhaka, Bangladesh

9. Dr. Arshadul Azim

Assistant Professor

Department of Nephrology

Shahid Sheikh Abu Naser Medical College

Hospital, Khulna, Bangladesh

10. Dr. Naushaba Tarannum Mahtab

Assistant Professor

Department of Obstetrics and Gynaecology

BIRDEM General Hospital, Dhaka, Bangladesh

11. Dr. Md. Rashedul Islam

Assistant Professor

Department of Neurology

BIRDEM General Hospital, Dhaka, Bangladesh

12. Dr.Mohammad Mehfuz-E-Khoda

Assistant Professor

Department of Nephrology

BIRDEM General Hospital, Dhaka, Bangladesh

13. Dr. Samira Rahat Afroze

Assistant Professor

Department of Internal Medicine

BIRDEM General Hospital, Dhaka, Bangladesh

14. Dr. Md. Jubaidul Islam

Registrar

Department of Internal Medicine

BIRDEM General Hospital, Dhaka, Bangladesh 